\title{
Higher Risk of Developing Peptic Ulcer Bleeding in Adult Patients Diagnosed with Hypoalbuminemia
}

\author{
Hsiao-Yun Hu ${ }^{1,6}$, Nicole Huang ${ }^{2}$ and Shen-Shong Chang ${ }^{1,3,4,5^{*}}$
}

${ }^{1}$ Institute of Public Health and Department of Public Health, Taipei City Hospital, Taipei, Taiwan

2 Institute of Hospital and Health Care Administration, Taipei City Hospital, Taipei, Taiwan

${ }^{3}$ School of Medicine; National Yang-Ming University, Taipei City Hospital, Taipei, Taiwan

${ }^{4}$ Division of Gastroenterology, Taipei City Hospital, Taipei, Taiwan

${ }^{5}$ Department of Internal Medicine; Taipei City Hospital Yang-Ming Branch, Taipei, Taiwan

${ }^{6}$ Department of Education and Research; Taipei City Hospital, Taipei, Taiwan

"Corresponding author: Shen Shong Chang, Division of Gastroenterology, Department of Internal Medicine, Taipei City Hospital Yang-Ming branch, No 105, Yusheng Street, Shihlin district, Taipei 111, Taiwan 11217, Tel: 886-2-28353456-6960; Fax: 886-2-28389551; E-mail: DAH20@tpech.gov.tw

Rec date: June 15, 2016; Acc date: Nov 9, 2016; Pub date: Nov 16, 2016

Copyright: ( $2016 \mathrm{Hu} \mathrm{HY}$, et al. This is an open-access article distributed under the terms of the Creative Commons Attribution License, which permits unrestricted use, distribution, and reproduction in any medium, provided the original author and source are credited.

\begin{abstract}
Aim: To evaluate whether patients diagnosed with hypoalbuminemia have higher risk of developing peptic ulcer bleeding (PUB).

Background: Low serum albumin levels are particularly common in critically ill patients. Life-threatening upper gastrointestinal (UGI) hemorrhage is a stress-related mucosal injury or stress ulceration in critically ill patients.

Patients and methods: We conducted a retrospective cohort study from the 2000-2010 database records of Taiwan's Bureau of National Health Insurance. Hypoalbuminemia patients were defined at least one of the inpatient claims with a diagnosis defined using ICD-9-CM diagnosis code: 273.8 . To be defined as a non-hypoalbuminemic, a person cannot have the code ICD-9-CM: 273.8 in their inpatient records or in the ambulatory care claims. The primary endpoint was hospitalization for PUB. We used a Cox's proportional-hazards model to estimate the risk of PUB in hypoalbuminemia patients.
\end{abstract}

Results: We enrolled 3,197 mild hypoalbuminemia patients, 490 severe hypoalbuminemia patients, and 14,748 non-hypoalbuminemia patients from 2000 to 2010. After adjusting for possible confounders, results indicated that the mild hypoalbuminemia group $(H R=1.75, p<0.001)$ and severe hypoalbuminemia $(H R=2.80, p<0.001)$ had a higher hospitalization rate for PUB, compared with the non-hypoalbuminemia group. Furthermore, non-steroidal antiinflammatory drugs use were critical risk factors for PUB in mild and severe hypoalbuminemia patients.

Conclusions: Patients diagnosed with hypoalbuminemia have a significantly elevated risk of developing PUB. Even though albumin normalizes after discharge, more attention should be paid to reduce the PUB risk in patients diagnosed with hypoalbuminemia.

Keywords: Albumin; Critical; Upper gastrointestinal hemorrhage; Peptic ulcer bleeding; Hypoalbuminemia; Non-steroidal antiinflammatory drugs

\section{Introduction}

Low serum albumin levels are particularly common in critically ill patients, reaching levels as high as $45-50 \%$ [1]. The hepatic synthesis of albumin is down-regulated in patients with a stress response, which is also commonly observed in critically ill patients [2]. Life-threatening upper gastrointestinal (UGI) hemorrhages are stress-related mucosal injuries or stress ulcerations in critically ill patients [3]. Terdiman et al., [4] reported a higher risk of rebleeding (39\%) and mortality (29\%) in unstable hospitalized patients.

Malnutrition and lower serum albumin levels affect peptic ulcer healing and cause peptic ulcer bleeding (PUB) and other complications [5]. Rady et al., [6] proposed that hypoalbuminemia patients receiving cardiac surgery were at a higher risk of developing PUB and facing lifethreatening episodes. Understanding the pathogenesis of PUB in hypoalbuminemia patients and seeking effective therapies is crucial in treating UGI hemorrhages to prevent life-threatening episodes.

Hypoalbuminemia in in acutely ill patients is related with poor outcomes and hospital stay is confirmed [7,8]. Population-based studies that have evaluated whether patients diagnosed with hypoalbuminemia are at an increased risk of developing PUB compared with the general population are scant. We divided participants into a mild, severe, and non-hypoalbuminemia groups. Drawing from the records of Taiwan's Bureau of National Health Insurance (NHI) database, we selected PUB patients who were entered into the database between 2000 and 2010. This study evaluates whether patients diagnosed with hypoalbuminemia is an independent factor that contributes to a higher risk of developing PUB compared with the 
general population and to identify the risk factors of PUB in hypoalbuminemia patients.

\section{Patients and Methods}

\section{Data source}

This nationwide cohort study was based on patient data obtained from the National Health Insurance Database (NHID), which is managed by Taiwan's National Health Research Institute (NHRI). The NHID contains health care data for $99 \%$ of Taiwan's population (approximately 23 million people) [9]. Sample files of the NHI Research Database (NHIRD) are composed of the comprehensive use and enrollment information for a randomly selected sample of 1000 $000 \mathrm{NHI}$ beneficiaries, representing approximately $5 \%$ of all enrollees in the year 2000. This study used the International Classifications of Diseases, Revision 9, and Clinical Modification (ICD-9-CM) to define diseases. This study was approved by the NHRI. The Institutional Review Board (IRB) of Taipei City Hospital approved this study (IRB No.: TCHIRB-1021104-E). Written consent was waived by the approving IRB.

\section{Participant selection}

We conducted a retrospective cohort study from January 1, 2000 to December 31, 2010, using ambulatory care and inpatient discharge records. Patients with prior gastrectomies and vagotomies were excluded. We excluded patients with diagnoses of gastric cancer and Zollinger-Ellison syndrome who were diagnosed between January 1, 1996 and the index date. Patients under the age of 20 years were excluded. We also excluded patients who had been hospitalized for PUB before their hypoalbuminemia episode. Patients who had been readmitted within 14 days of discharge from the date of hospitalization for their hypoalbuminemia episode were also excluded. Figure 1 shows the flow of hypoalbuminemia patients during the course of this study.

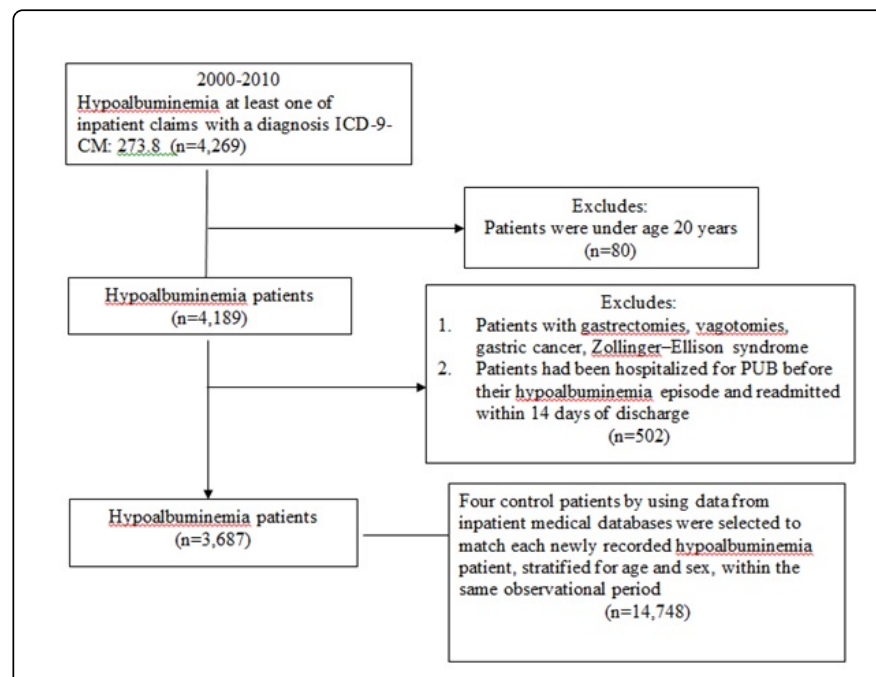

Figure 1: Flowchart depicting the selection of the participants.

According to the reimbursement policy of the NHI, patients with serum albumin level less than $2.5 \mathrm{~g}$ per deciliter are reimbursed for albumin therapy. Therefore, severe hypoalbuminemia patients were defined using the ICD-9-CM diagnosis code: 273.8 at least one of inpatient claims (including intensive care unit stay) and receiving albumin therapy. Mild hypoalbuminemia patients were defined using the ICD-9-CM diagnosis code: 273.8 at least one of inpatient claims, but excluding severe hypoalbuminemia patients. To be defined as nonhypoalbuminemic, a patient cannot have the code ICD-9-CM: 273.8 in their inpatient records or in the ambulatory care claims. Four control patients by using data from inpatient medical databases were selected to match each newly recorded hypoalbuminemia patient by random sampling, stratified for age and sex, from the database within the same observational period.

\section{Definition of Helicobacter pylori associated peptic ulcer}

We used ICD-9-CM code (531.xx, 532.xx, or 533.xx) after the endoscopic examination to identify peptic ulcer patients. The diagnosis of Helicobacter pylori status in our study was performed using rapid urease tests (RUT) or a histological assessment using hematoxylin and eosin staining. Enrolled patients who received treatment for the eradication of $H$. pylori during the 11 year period were defined as having $H$. pylori associated peptic ulcers. $H$. pylori eradication therapy was defined as using triple or quadruple therapy consisting of proton pump inhibitors (PPIs) or histamine receptor-2 blockers (H2blockers), clarithromycin or tetracycline, amoxicillin or metronidazole, and bismuth or no bismuth [10].

\section{Patient characteristics}

Conditions that required inpatient care or 3 or more ambulatorycare visits between January 1, 1996, and the index date of our study were defined as comorbidities. The comorbidities identified in our cohort and the corresponding ICD-9-CM diagnosis codes were as follows: diabetes mellitus (DM; ICD-9-CM 250), congestive heart failure (CHF; ICD-9-CM 428), coronary artery disease (CAD; ICD-9CM 410-414), liver cirrhosis (ICD-9-CM 571.2, 571.5, and 571.6), nephrotic syndrome (ICD-9-CM 581), malignancy (ICD-9-CM 140-208), and dyslipidemia (ICD-9-CM 272-274).

We recorded the age and sex of the patients. Patients were defined as users of PPIs, aspirin, non-steroidal anti-inflammatory drugs (NSAIDs), cyclooxygenase-2 (COX-2) specific inhibitors, steroids, and calcium channel blockers based on whether they had used at least one prescription of the respective medication within 28 days of the claim date of hospitalization for PUB. Subjects without PUB will capture the whole follow-up period.

\section{End point}

The primary endpoint of this study was hospitalization for PUB, as defined by ICD-9-CM 531.0, 531.2, 531.4, 531.6, 532.0, 532.2, 532.4, 532.6, 533.0, 533.2, 533.4, and 533.6, following endoscopic examination after a hypoalbuminemia episode, between 2000 and 2010, based on inpatient discharge records. Severe PUB was defined by ICD-9-CM code and receiving endoscopic hemostasis and surgical intervention. Each patient case was followed until the outcome or until the end of the study period.

\section{Statistical analysis}

Demographic data were expressed as categorical data and mean \pm SD. $\chi^{2}$ Test was used for categorical data. Risk estimates, presented as the hazard ratio (HR) and 95\% confidence intervals (CI), were also calculated to determine if hypoalbuminemia was independently 
Citation: Hu HY, Huang N,Chang SS (2017) Higher Risk of Developing Peptic Ulcer Bleeding in Adult Patients Diagnosed with Hypoalbuminemia. Gen Med (Los Angeles) 4: 277. doi:10.4172/2327-5146.1000277

Page 3 of 8

associated with an increased risk of developing PUB. Survival analysis was assessed using the Kaplan-Meier analysis, with significance according to log-rank test. Survival time (freedom from PUB) was calculated from the date of enrollment to the date of hospitalization for PUB. A P value of less than 0.05 was considered significant. All statistical analyses were performed using the SAS System for Windows, version 9.3 (SAS Institute, Cary, NC, USA).

\section{Results}

We enrolled 3,197 mild hypoalbuminemia patients, 490 severe hypoalbuminemia patients, and 14,748 non-hypoalbuminemia patients from 2000 to 2010 . Table 1 displays demographic data, including age, sex, comorbidities; H. pylori associated peptic ulcer, and medications.

\begin{tabular}{|c|c|c|c|c|c|c|c|}
\hline \multirow[t]{3}{*}{ Variables } & \multirow{2}{*}{\multicolumn{2}{|c|}{ Control }} & \multicolumn{4}{|c|}{ Hypoalbuminemia } & \multirow[t]{3}{*}{ P-value } \\
\hline & & & \multicolumn{2}{|l|}{ Mild } & \multicolumn{2}{|l|}{ Severe } & \\
\hline & $n=14,748$ & $\%$ & $n=3,197$ & $\%$ & $\mathrm{n}=490$ & $\%$ & \\
\hline \multicolumn{8}{|l|}{ Age } \\
\hline $20-49$ & 2,256 & 15.3 & 498 & 15.58 & 66 & 13.47 & 0.804 \\
\hline $50-69$ & 3,988 & 27.04 & 858 & 26.84 & 139 & 28.37 & \\
\hline$\geq 70$ & 8,504 & 57.66 & 1,841 & 57.59 & 285 & 58.16 & \\
\hline \multicolumn{8}{|l|}{ Sex } \\
\hline Male & 8,436 & 57.2 & 1,817 & 56.83 & 292 & 59.59 & 0.517 \\
\hline Female & 6,312 & 42.8 & 1,380 & 43.17 & 198 & 40.41 & \\
\hline \multicolumn{8}{|l|}{ Comorbidities } \\
\hline DM & 2,548 & 17.28 & 519 & 16.23 & 74 & 15.1 & 0.187 \\
\hline $\mathrm{CHF}$ & 1,886 & 12.79 & 432 & 13.51 & 49 & 10 & 0.088 \\
\hline CAD & 3,506 & 23.77 & 638 & 19.96 & 65 & 13.27 & $<0.001$ \\
\hline Liver cirrhosis & 514 & 3.49 & 341 & 10.67 & 89 & 18.16 & $<0.001$ \\
\hline Nephrotic syndrome & 141 & 0.96 & 72 & 2.25 & 27 & 5.51 & $<0.001$ \\
\hline Malignancy & 2,545 & 17.26 & 684 & 21.4 & 86 & 17.55 & $<0.001$ \\
\hline Dyslipidemia & 3,002 & 20.36 & 484 & 15.14 & 40 & 8.16 & $<0.001$ \\
\hline H. pylori associated peptic ulcer & 1,285 & 8.71 & 251 & 7.85 & 30 & 6.12 & 0.046 \\
\hline \multicolumn{8}{|l|}{ Medications } \\
\hline PPIs & 372 & 2.52 & 109 & 3.41 & 17 & 3.47 & 0.011 \\
\hline Aspirin & 1,363 & 9.24 & 196 & 6.13 & 18 & 3.67 & $<0.001$ \\
\hline NSAIDs & 2,227 & 15.1 & 368 & 11.51 & 38 & 7.76 & $<0.001$ \\
\hline COX-2 inhibitors & 626 & 4.24 & 84 & 2.63 & 12 & 2.45 & $<0.001$ \\
\hline Steroid & 889 & 6.03 & 218 & 6.82 & 21 & 4.29 & 0.055 \\
\hline Calcium channel blockers & 2,163 & 14.67 & 327 & 10.23 & 35 & 7.14 & $<0.001$ \\
\hline Peptic ulcer bleeding & 617 & 4.18 & 153 & 4.79 & 28 & 5.71 & 0.099 \\
\hline Follow-up year (mean $\pm S D$ ) & \multicolumn{2}{|l|}{$3.74 \pm 3.19$} & \multicolumn{2}{|c|}{$2.44 \pm 2.84$} & \multicolumn{2}{|c|}{$1.66 \pm 2.41$} & \\
\hline
\end{tabular}

Table 1: Demographic characteristics of hypoalbuminemia cohort and matched control Definition of Hypoalbuminemia and Nonhypoalbuminemia Group. 
Citation: Hu HY, Huang N,Chang SS (2017) Higher Risk of Developing Peptic Ulcer Bleeding in Adult Patients Diagnosed with Hypoalbuminemia. Gen Med (Los Angeles) 4: 277. doi:10.4172/2327-5146.1000277

Page 4 of 8

\section{Hypoalbuminemia as an Independent risk factor for PUB}

After adjusting for age, sex, comorbidities, H. pylori associated peptic ulcer, and medications, the results from Cox's proportional hazards model analysis indicated that the mild hypoalbuminemia group $(\mathrm{HR}=1.75, \mathrm{p}<0.001)$ and severe hypoalbuminemia group
$(\mathrm{HR}=2.80, \mathrm{p}<0.001)$ had a higher hospitalization rate for PUB, compared with the non-hypoalbuminemia group (Table 2). The logrank test and Kaplan-Meier survival analysis showed that severe and mild hypoalbuminemia patients had significantly higher rate of PUB than control group, respectively (all $\mathrm{p}<0.001$ ) (Figure 2 ).

\begin{tabular}{|c|c|c|c|c|c|c|}
\hline \multirow[t]{2}{*}{ Variables } & \multicolumn{3}{|c|}{ Univariate } & \multicolumn{3}{|c|}{ Multivariate } \\
\hline & HR & $95 \% \mathrm{Cl}$ & P-value & HR & $95 \% \mathrm{Cl}$ & P-value \\
\hline Mild vs. control & 1.59 & $1.33-1.89$ & $<0.001$ & 1.75 & $1.46-2.10$ & $<0.001$ \\
\hline Severe vs. control & 2.49 & $1.70-3.63$ & $<0.001$ & 2.8 & $1.90-4.12$ & $<0.001$ \\
\hline \multicolumn{7}{|l|}{ Age } \\
\hline $50-69$ vs. $20-49$ & 0.72 & $0.61-0.85$ & $<0.001$ & 1.78 & $1.34-2.36$ & $<0.001$ \\
\hline$\geq 70$ vs. $20-49$ & 2.21 & $1.90-2.57$ & $<0.001$ & 3.41 & $2.61-4.44$ & $<0.001$ \\
\hline Male vs. Female & 1.35 & $1.17-1.56$ & $<0.001$ & 1.36 & $1.17-1.57$ & $<0.001$ \\
\hline \multicolumn{7}{|l|}{ Comorbidities } \\
\hline DM & 1.2 & $1.01-1.42$ & 0.035 & 1.11 & $0.93-1.32$ & 0.234 \\
\hline $\mathrm{CHF}$ & 1.67 & $1.40-1.99$ & $<0.001$ & 1.21 & $1.00-1.45$ & 0.048 \\
\hline CAD & 1.2 & $1.03-1.40$ & 0.023 & 0.85 & $0.72-1.00$ & 0.05 \\
\hline Liver cirrhosis & 2.5 & $1.97-3.17$ & $<0.001$ & 2.46 & $1.92-3.15$ & $<0.001$ \\
\hline Nephrotic syndrome & 0.83 & $0.43-1.61$ & 0.586 & 0.65 & $0.34-1.26$ & 0.202 \\
\hline Malignancy & 0.8 & $0.64-0.99$ & 0.044 & 0.67 & $0.54-0.84$ & $<0.001$ \\
\hline Dyslipidemia & 0.76 & $0.63-0.91$ & 0.002 & 0.69 & $0.57-0.83$ & $<0.001$ \\
\hline H. pylori associated peptic ulcer & 1.83 & $1.51-2.21$ & $<0.001$ & 1.69 & $1.40-2.06$ & $<0.001$ \\
\hline \multicolumn{7}{|l|}{ Medications } \\
\hline PPIs & 2.47 & $1.84-3.33$ & $<0.001$ & 1.69 & $1.25-2.29$ & $<0.001$ \\
\hline Aspirin & 2.72 & $2.29-3.24$ & $<0.001$ & 1.98 & $1.64-2.39$ & $<0.001$ \\
\hline NSAIDs & 3.02 & $2.61-3.50$ & $<0.001$ & 2.88 & $2.48-3.35$ & $<0.001$ \\
\hline COX-2 inhibitors & 3.05 & $2.43-3.82$ & $<0.001$ & 2.02 & $1.60-2.55$ & $<0.001$ \\
\hline Steroid & 1.93 & $1.53-2.43$ & $<0.001$ & 1.34 & $1.06-1.70$ & 0.015 \\
\hline Calcium channel blockers & 2.09 & $1.78-2.45$ & $<0.001$ & 1.34 & $1.12-1.59$ & 0.001 \\
\hline
\end{tabular}

Table 2: Multivariate Cox proportional hazards model analysis for predicting of peptic ulcer bleeding. 
Citation: Hu HY, Huang N,Chang SS (2017) Higher Risk of Developing Peptic Ulcer Bleeding in Adult Patients Diagnosed with Hypoalbuminemia. Gen Med (Los Angeles) 4: 277. doi:10.4172/2327-5146.1000277

Page 5 of 8

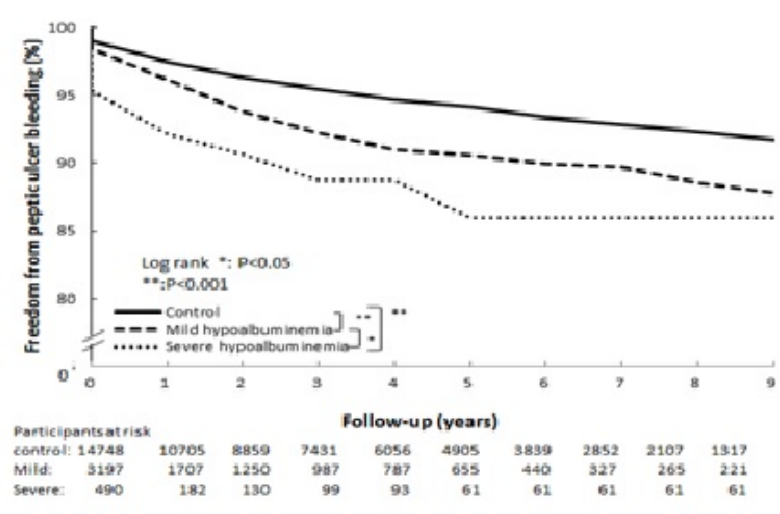

Figure 2: Kaplan-Meier estimates of survival free of peptic ulcer bleeding in subjects categorized by severe hypoalbuminemia, mild hypoalbuminemia, and non-hypoalbuminemia. The event-free survival rates were significantly different between severe hypoalbuminemia and mild hypoalbuminemia $(\mathrm{p}<0.05$ by log rank test) and between severe hypoalbuminemia and control group $(\mathrm{p}<0.001$ by log rank test) and between mild hypoalbuminemia and control group $(\mathrm{p}<0.001$ by log rank test).

\section{Risk factors of PUB in hypoalbuminemia patients}

Cox proportional hazards model analysis indicated that CHF, liver cirrhosis, $H$. pylori associated peptic ulcer, aspirin use, and NSAIDs use were critical risk factors for PUB in mild hypoalbuminemia patients (Table 3). Table 4 showed that NSAIDs use was critical risk factor for PUB in severe hypoalbuminemia patients. Moreover, hypoalbuminemia patients had significantly higher rate of severe PUB requiring endoscopic hemostasis and surgical intervention $(\mathrm{HR}=1.65$, $\mathrm{p}=0.006$ ), compared with the general population (data not shown).

\section{Multivariate stratified analysis}

Figure 3 showed the result of multivariate stratified analysis in mild hypoalbuminemia patients. Each factor was adjusted for all other factors including age, sex, comorbidities, $H$. pylori associated peptic ulcer, and medications as in Table 1 . For patients without $\mathrm{CHF}$ $(\mathrm{HR}=1.66, \mathrm{p}<0.001)$, without liver cirrhosis $(\mathrm{HR}=1.81, \mathrm{p}<0.001)$, and without $H$. pylori associated peptic ulcer $(H R=1.74, \mathrm{p}<0.001)$, mild hypoalbuminemia was associated with a higher risk of PUB. For patients not using aspirin $(\mathrm{HR}=1.75, \mathrm{p}<0.001)$ and not using NSAIDs $(\mathrm{HR}=1.85, \mathrm{p}<0.001)$ constituted significantly higher risks of developing PUB in mild hypoalbuminemia patients. Moreover, for patients without $\mathrm{CHF}(\mathrm{HR}=2.76, \mathrm{p}<0.001)$, without liver cirrhosis $(\mathrm{HR}=2.88, \mathrm{p}<0.001)$, and without $H$. pylori associated peptic ulcer $(\mathrm{HR}=2.89, \mathrm{p}<0.001)$, severe hypoalbuminemia was associated with a higher risk of PUB. For patients not using aspirin $(\mathrm{HR}=2.88, \mathrm{p}<0.001)$ and not using NSAIDs $(\mathrm{HR}=2.22, \mathrm{p}=0.001)$ constituted significantly higher risks of developing PUB in severe hypoalbuminemia patients (Figure 4).

\begin{tabular}{|c|c|c|c|c|c|c|}
\hline \multirow[t]{2}{*}{ Variables } & \multicolumn{3}{|c|}{ Univariate } & \multicolumn{3}{|c|}{ Multivariate } \\
\hline & HR & $95 \% \mathrm{Cl}$ & P-value & HR & $95 \% \mathrm{Cl}$ & P-value \\
\hline \multicolumn{7}{|l|}{ Age } \\
\hline $50-69$ vs. $20-49$ & 0.85 & $0.59-1.22$ & 0.37 & 1.08 & $0.64-1.80$ & 0.778 \\
\hline$\geq 70$ vs. $20-49$ & 1.47 & $1.07-2.03$ & 0.019 & 1.59 & $0.99-2.55$ & 0.055 \\
\hline Male vs. female & 1.28 & $0.92-1.77$ & 0.137 & 1.26 & $0.91-1.76$ & 0.161 \\
\hline \multicolumn{7}{|l|}{ Comorbidities } \\
\hline DM & 0.89 & $0.59-1.36$ & 0.603 & 0.86 & $0.55-1.32$ & 0.482 \\
\hline $\mathrm{CHF}$ & 1.73 & $1.19-2.53$ & 0.004 & 1.55 & $1.03-2.35$ & 0.037 \\
\hline CAD & 1.08 & $0.75-1.56$ & 0.686 & 0.79 & $0.53-1.19$ & 0.266 \\
\hline Liver cirrhosis & 1.65 & $1.05-2.57$ & 0.028 & 1.94 & $1.21-3.12$ & 0.006 \\
\hline Nephrotic syndrome & 0.22 & $0.03-1.60$ & 0.135 & 0.25 & $0.03-1.79$ & 0.167 \\
\hline Malignancy & 0.81 & $0.51-1.30$ & 0.391 & 0.71 & $0.44-1.15$ & 0.163 \\
\hline Dyslipidemia & 0.8 & $0.52-1.23$ & 0.308 & 0.8 & $0.51-1.26$ & 0.336 \\
\hline H. pylori associated peptic ulcer & 1.75 & $1.12-2.75$ & 0.015 & 1.7 & $1.07-2.70$ & 0.025 \\
\hline \multicolumn{7}{|l|}{ Medications } \\
\hline PPIs & 2.47 & $1.34-4.56$ & 0.004 & 1.89 & $0.99-3.59$ & 0.052 \\
\hline
\end{tabular}


Citation: Hu HY, Huang N,Chang SS (2017) Higher Risk of Developing Peptic Ulcer Bleeding in Adult Patients Diagnosed with Hypoalbuminemia. Gen Med (Los Angeles) 4: 277. doi:10.4172/2327-5146.1000277

Page 6 of 8

\begin{tabular}{|l|l|l|l|l|l|l|}
\hline Aspirin & 2.41 & $1.52-3.82$ & $<0.001$ & 2.02 & $1.23-3.34$ & 0.006 \\
\hline NSAIDs & 2.66 & $1.86-3.82$ & $<0.001$ & 2.43 & $1.66-3.56$ & $<0.001$ \\
\hline COX-2 inhibitors & 2.46 & $1.29-4.67$ & 0.006 & 1.28 & $0.64-2.57$ & 0.491 \\
\hline Steroid & 1.54 & $0.91-2.63$ & 0.11 & 1.19 & $0.68-2.07$ & 0.549 \\
\hline Calcium channel blockers & 1.98 & $1.33-2.95$ & $<0.001$ & 1.39 & $0.88-2.18$ & 0.156 \\
\hline
\end{tabular}

Table 3: Multivariate Cox proportional hazards model analysis for predicting of peptic ulcer bleeding in mild hypoalbuminemia patients.

\begin{tabular}{|c|c|c|c|c|c|c|}
\hline \multirow[t]{2}{*}{ Variables } & \multicolumn{3}{|c|}{ Univariate } & \multicolumn{3}{|c|}{ Multivariate } \\
\hline & HR & $95 \% \mathrm{Cl}$ & P-value & HR & $95 \% \mathrm{Cl}$ & P-value \\
\hline \multicolumn{7}{|l|}{ Age } \\
\hline $50-69$ vs. $20-49$ & 0.74 & $0.31-1.74$ & 0.49 & 0.64 & $0.22-1.90$ & 0.42 \\
\hline$\geq 70$ vs. $20-49$ & 0.89 & $0.42-1.87$ & 0.753 & 0.5 & $0.17-1.51$ & 0.222 \\
\hline Male vs. female & 1.63 & $0.72-3.71$ & 0.241 & 2.05 & $0.83-5.02$ & 0.119 \\
\hline \multicolumn{7}{|l|}{ Comorbidities } \\
\hline DM & 0.35 & $0.08-1.48$ & 0.154 & 0.28 & $0.06-1.32$ & 0.108 \\
\hline $\mathrm{CHF}$ & 1.38 & $0.48-3.98$ & 0.553 & 1.59 & $0.41-6.17$ & 0.504 \\
\hline CAD & 1.45 & $0.55-3.81$ & 0.453 & 1.62 & $0.50-5.25$ & 0.422 \\
\hline Liver cirrhosis & 1.55 & $0.68-3.54$ & 0.294 & 1.4 & $0.53-3.72$ & 0.502 \\
\hline Nephrotic syndrome & 0.36 & $0.05-2.69$ & 0.321 & 0.28 & $0.03-2.32$ & 0.239 \\
\hline Malignancy & 1.39 & $0.52-3.66$ & 0.51 & 1.4 & $0.49-4.02$ & 0.534 \\
\hline Dyslipidemia & 0.56 & $0.13-2.35$ & 0.424 & 1.08 & $0.21-5.55$ & 0.923 \\
\hline H. pylori associated peptic ulcer & 2.5 & $0.86-7.21$ & 0.091 & 1.41 & $0.42-4.74$ & 0.58 \\
\hline \multicolumn{7}{|l|}{ Medications } \\
\hline PPls & 0.93 & $0.13-6.84$ & 0.942 & 0.69 & $0.08-5.80$ & 0.736 \\
\hline Aspirin & 1.04 & $0.14-7.68$ & 0.967 & 1.58 & $0.17-14.61$ & 0.685 \\
\hline NSAIDs & 6.55 & $3.02-14.2$ & $<.0001$ & 6.01 & $2.47-14.64$ & $<0.001$ \\
\hline COX-2 inhibitors & 4.02 & $0.95-16.95$ & 0.059 & 4.14 & $0.72-23.77$ & 0.112 \\
\hline Steroid & 0.75 & $0.10-5.49$ & 0.774 & 0.65 & $0.08-5.51$ & 0.696 \\
\hline Calcium channel blockers & 1.13 & $0.34-3.75$ & 0.844 & 1.04 & $0.26-4.21$ & 0.96 \\
\hline
\end{tabular}

Table 4: Multivariate Cox proportional hazards model analysis for predicting of peptic ulcer bleeding in severe hypoalbuminemia patients.

\section{Discussion}

Our data showed that patients diagnosed with hypoalbuminemia have a significantly higher risk of developing PUB. Compared with the general population, patients with severe hypoalbuminemia history have a 3-fold increased risk of developing PUB in our study.

Tung et al., [11] conducted a retrospective study in a single tertiary hospital with a chart record and disclosed that hypoalbuminemia is common in patients with non-variceal UGI hemorrhage. The outcome is a UGI hemorrhage, which includes hematemesis, gross blood or coffee grounds material, hematochezia, and melena. The result is similar to ours. However, the methodology of our study is different from that of Tung et al, which enrolled patients with hypoalbuminemia, emergency room visits, hospital-based status, no record of ulcerogenic medication, and shorter duration follow-up (12 months). However, our methods were used to analyze patients diagnosed with hypoalbuminemia who were nationally-based, conduct a long-term follow-up, evaluate ulcerogenic medications, and conduct an 11 year-long longitudinal study, and PUB for hospitalization. 
Citation: Hu HY, Huang N,Chang SS (2017) Higher Risk of Developing Peptic Ulcer Bleeding in Adult Patients Diagnosed with Hypoalbuminemia. Gen Med (Los Angeles) 4: 277. doi:10.4172/2327-5146.1000277

Page 7 of 8

Cheng et al., [5] conducted a study and disclosed that hypoalbuminemia with comorbid illness has a higher risk of developing a 7-day and a 28-day recurrent PUB. The intra-stomach acid environment is crucial for the acute stage of ulcer healing. Malnourished patients with more comorbidities have a higher mortality rate from UGI hemorrhage because of poor ulcer healing $[3,12]$. Therefore, the intravenous PPIs dosage and duration were the predictors of peptic ulcer rebleeding in hypoalbuminemia patients. In contrast with the study of Cheng et al., our 11-year longitudinal study analyzed hypoalbuminemia patients during the follow-up and evaluated ulcerogenic medications in a larger cohort, and PUB for hospitalization.

Physicians who care for long-term follow-up hypoalbuminemia patients should be aware of the predictors and potentially severe complications. The increased production of the reactive oxygen radicals in the antrum and duodenum was found to be related to antral H. pylori density and associated with duodenal ulceration [13]. Albumin is an essential binding protein, which acts as an extracellular scavenger in response to oxidative stress [14]. Therefore, the $H$. pylori eradication failure rate in hypoalbuminemia patients is a critical issue. Performing a prospective randomized controlled study to evaluate whether $H$. pylori eradication therapy can cause a drastic reduction of PUB episodes in an hypoalbuminemia state, similarly to in the general population, is necessary.

\begin{tabular}{|c|c|c|c|}
\hline Subgroup & Number & & Hazard Ratio ( $95 \%$ C.I) \\
\hline \multicolumn{4}{|l|}{ Age } \\
\hline $20-49$ & 2,754 & & $-2.97(1.65-5.33)$ \\
\hline 50.69 & 4,846 & 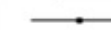 & $1.92(1.34-2.76)$ \\
\hline$\geq 70$ & 10,345 & 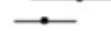 & $1.49(1.18-1.88)$ \\
\hline \multicolumn{4}{|r|}{$1.7,(1,100,00)$} \\
\hline Male & 10,253 & 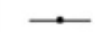 & $1.66(1.32-2.09)$ \\
\hline \multirow{2}{*}{\multicolumn{4}{|c|}{ CHF }} \\
\hline & & & \\
\hline Yes & 2,318 & & $2.11(1.43-3.10)$ \\
\hline No & 15,627 & $\rightarrow$ & $1.66(1.36-2.04)$ \\
\hline \multicolumn{4}{|c|}{ Liver cirrhosis } \\
\hline Yes & 855 & - & $1.02(0.60-1.74)$ \\
\hline No & 17,090 & $\rightarrow$ & $1.81(1.49-2.19)$ \\
\hline \multicolumn{4}{|c|}{ Malignancy } \\
\hline Yes & 3,229 & & $1.64(0.98-2.75)$ \\
\hline \multirow{2}{*}{\multicolumn{4}{|c|}{ Desslipidemia }} \\
\hline & & & \\
\hline Yes & 3,486 & 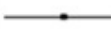 & $1.72(1.10-2.69)$ \\
\hline No & 14,459 & 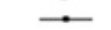 & $1.74(1.43-2.12)$ \\
\hline \multicolumn{4}{|l|}{ H. pylori } \\
\hline Yes & 1,536 & 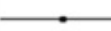 & $1.71(1.06-2.77)$ \\
\hline No & 16,409 & $\because$ & $1.74(1.43-2.11)$ \\
\hline \multicolumn{4}{|l|}{ PPIs } \\
\hline Yes & 481 & & $1.74(0.83-3.64)$ \\
\hline $\begin{array}{c}\text { No } \\
\text { Aspirin }\end{array}$ & 17,464 & $\rightarrow$ & $1.76(1.46-2.12)$ \\
\hline \multicolumn{4}{|l|}{ Aspirin } \\
\hline Yes & 1,559 & $\longrightarrow$ & $1.71(1.07-2.74)$ \\
\hline No & 16,386 & $\rightarrow$ & $1.75(1.44-2.13)$ \\
\hline \multicolumn{4}{|l|}{ NSAIDs } \\
\hline & 2,595 & 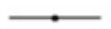 & $1.60(1.14-2.25)$ \\
\hline No & 15,350 & $\longrightarrow$ & $1.85(1.49-2.29)$ \\
\hline \multicolumn{4}{|c|}{ COX-2 inhibitors } \\
\hline Yes & 710 & — & $1.06(0.52-2.16)$ \\
\hline & 17,235 & $\longrightarrow$ & $1.85(1.53-2.24)$ \\
\hline \multicolumn{4}{|c|}{ Calcium channel blockers } \\
\hline Yes & 2,490 &. & $1.71(1.15-2.53)$ \\
\hline No & 15,455 & $\longrightarrow$ & $1.78(1.45-2.18)$ \\
\hline & & 2 & 8 \\
\hline
\end{tabular}

Figure 3: Multivariate stratified analysis: Adjusted hazard ratios for peptic ulcer bleeding in mild hypoalbuminemia patients (Each factor was adjusted for all other factors).

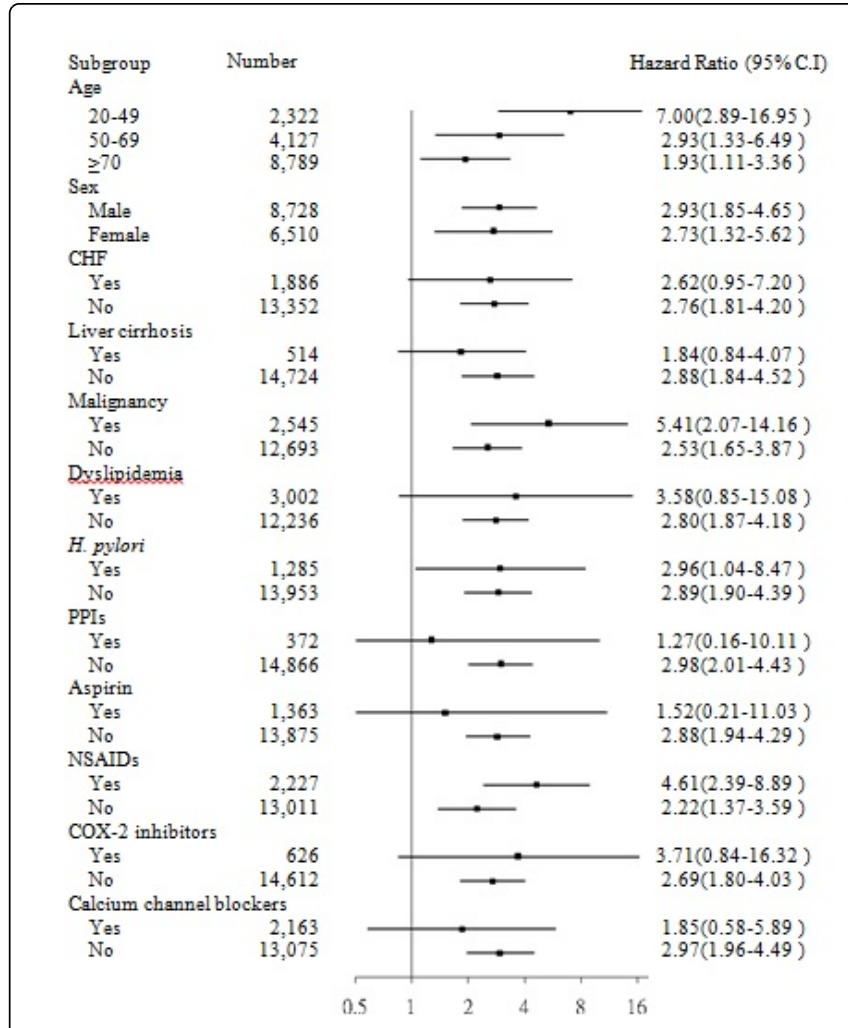

Figure 4: Multivariate stratified analysis: Adjusted hazard ratios for peptic ulcer bleeding in sever hypoalbuminemia patients (Each factor was adjusted for all other factors).

The use of ulcerogenic medication constitutes a critical risk factor for PUB [15,16]. This study showed that severe and mild hypoalbuminemia patients have a low probability of using aspirin (3.67\% vs. $6.13 \%$ vs. $9.24 \%$ ), NSAIDs (7.76\% vs. $11.51 \%$ vs. $15.10 \%)$, and COX-2 inhibitors ( $2.45 \%$ vs. $2.63 \%$ vs. $4.24 \%)$, compared to the general population (Table 1). A stratified analysis according to ulcerogenic agents revealed a higher risk of developing PUB in mild and severe hypoalbuminemia patients who are not using aspirin, NSAIDs, or COX-2 specific inhibitors (Figures 3 and 4). Therefore, hypoalbuminemia per se is an independent risk factor for PUB, irrespective of the ulcerogenic medications.

Our results showed that severe and mild hypoalbuminemia patients are less likely to use ulcerogenic medication, such as aspirin, NSAIDs, and COX-2 inhibitors, and fewer $\mathrm{H}$. pylori associated with peptic ulcer $(6.12 \%$ vs. $7.85 \%$ vs. $8.71 \%)$, compared to non-hypoalbuminemia patients (Table 1). Further study is required to confirm whether hypoalbuminemia patients have a higher risk of developing non- $H$. pylori and non-NSAID peptic ulcers, as do other comorbidities, such as liver cirrhosis, malignancy, and ESRD [17-19].

Our study noted several limitations. First, hypoalbuminemia severity is considered the most powerful prognostic factor because malnutrition is a consequence of poor ulcer healing [3]. However, certain laboratory data such as the level of serum albumin was not available in the NHIRD. Therefore, we used the ICD-9CM code: 273.8 and albumin therapy to classify mild and severe hypoalbuminemia. We ascertained the occurrence of hypoalbuminemia by investigating only 
hospitalized patients, whose ICD coding were strictly audited for the purpose of reimbursement. The Bureau of the NHI has randomly sample the claim data from every hospital and review charts to verify the diagnostic validity. Moreover, establishing a standard definition of the endpoint is crucial; therefore, we analyzed only the risk of hospitalization for PUB by using data from inpatient medical databases. Second, prophylactic acid suppression therapy with PPIs might reduce the risk of PUB in critically ill patients [20]. However, PPIs are lower in cost: most cost less than US $\$ 0.8$ per tablet. Therefore, participants in current study were receiving prophylactic PPIs within 28 days of the claim date for PUB. More patients in the severe and mild hypoalbuminemia groups were receiving PPIs $(3.47 \%$ vs. $3.41 \%$ vs. $2.52 \%, \mathrm{p}=0.011$ ), compared to the non-hypoalbuminemia group. Therefore, it likely does not bias the results and toward to null. Third, smoking and drinking, which are related risk factors of PUB, were not available in the database. Fourth, our database lacks information regarding the presence of $H$. pylori [21] and we use $H$. pylori associated with peptic ulcers as surrogate. Our results showed that severe and mild hypoalbuminemia patients are fewer $H$. pylori associated with peptic ulcer $(6.12 \%$ vs. $7.85 \%$ vs. $8.71 \%)$, compared to non-hypoalbuminemia patients. Therefore, it will be non-differential misclassification and bias towards to null. Finally, the hypoalbuminemic status might not be constant in the same subject. Because the hypoalbuminemia status might improve after they recover from an acute illness that caused their hospitalization. Therefore, we adopt a strict definition of non-hypoalbuminemic, a patient cannot have the code ICD-9-CM: 273.8 in their inpatient records or in the ambulatory care claims between 2000 and 2010, compared to mild and severe hypoalbuminemia.

In conclusion, patients diagnosed with hypoalbuminemia have a significantly higher risk of developing PUB. Even though albumins normalize after discharge, more attention should be paid to reduce the PUB risk in patients diagnosed with hypoalbuminemia.

\section{Acknowledgements}

We wish to thank the Taiwan Ministry of Education for their support for this work through its "Aim for the Top University Plan." We would like to thank the Bureau of National Health Insurance and the NHRI for making the NHIRD available to us for this study. We also thank the anonymous reviewers and editors for their comments.This work was supported by grand number 10401-62-067 from Taipei City Hospital, Taiwan

\section{Disclosures and Competing Interests}

No author has conflicts of interest or financial arrangements that could potentially influence the described.

\section{References}

1. Ulldemolins M, Roberts JA, Paterson DL, Lipman J (2011) The effects of hypoalbuminaemia on optimizing antibacterial dosing in critically ill patients. Clin Pharmacokinet 50: 99-110.

2. Kushner I (1982) The phenomenon of the acute phase response. Ann N Y Acad Sci 389: 39-48.
3. Cook DJ, Fuller HD, Guyatt GH, Marshall JC, Leasa D, et al. (1994) Risk factors for gastrointestinal bleeding in critically ill patients. Canadian Critical Care Trials Group. N Engl J Med 330: 377-381.

4. Terdiman JP, Ostroff JW (1998) Gastrointestinal bleeding in the hospitalized patient: a case-control study to assess risk factors, causes, and outcome. Am J Med 104: 349-354.

5. Cheng HC, Kao AW, Chuang CH, Sheu BS (2005) The efficacy of highand low-dose intravenous omeprazole in preventing rebleeding for patients with bleeding peptic ulcers and comorbid illnesses. Dig Dis Sci 50: 1194-1201.

6. Rady MY, Ryan T, Starr NJ (1997) Clinical characteristics of preoperative hypoalbuminemia predict outcome of cardiovascular surgery. J Parenter Enteral Nutr 21: 81-90.

7. Reinhardt GF, Myscofski JW, Wilkens DB, Dobrin PB, Mangan JE Jr, et al. (1980) Incidence and mortality of hypoalbuminemic patients in hospitalized veterans. JPEN J Parenter Enteral Nutr. 4:357-359.

8. Vincent JL, Dubois MJ, Navickis RJ, Wilkes MM (2003) Hypoalbuminemia in acute illness: is there a rationale for intervention? A meta-analysis of cohort studies and controlled trials. Ann Surg. 237:319-334.

9. Cheng SH, Chiang TL (1997) The effect of universal health insurance on health care utilization in Taiwan. Results from a natural experiment. JAMA 278:89-93.

10. Hsiao FY, Tsai YW, Wen YW, Kuo KN, Tsai CR, et al. (2011) Effect of Helicobacter pylori eradication therapy on risk of hospitalization for a major ulcer event. Pharmacotherapy 31: 239-247.

11. Tung CF, Chow WK, Chang CS, Peng YC, Hu WH (2007) The prevalence and significance of hypoalbuminemia in non-variceal upper gastrointestinal bleeding. Hepatogastroenterology 54: 1153-1156.

12. Cheng HC, Chuang SA, Kao YH, Kao AW, Chuang CH, et al. (2003) Increased risk of rebleeding of peptic ulcer bleeding in patients with comorbid illness receiving omeprazole infusion. Hepatogastroenterology 2003; 2270-2273.

13. Zhang Q, Dawodu JB, Etolhi G, Husain A, Gemmell CG, et al. (1997) Relationship between the mucosal production of reactive oxygen radicals and density of Helicobacter pylori in patients with duodenal ulcer. Eur J Gastroenterol Hepatol 9: 261-265.

14. Soeters PB (2009) Rationale for albumin infusions. Curr Opin Clin Nutr Metab Care 12: 258-264.

15. Huang JQ, Sridhar S, Hunt RH (2002) Role of Helicobacter pylori infection and non-steroidal anti-inflammatory drugs in peptic-ulcer disease: a meta-analysis. Lancet 359: 14-22.

16. Hsiang KW, Chen TS, Lin HY, Luo JC, Lu CL, et al. (2010) Incidence and possible risk factors for clinical upper gastrointestinal events in patients taking selective cyclooxygenase-2 inhibitors: A prospective, observational, cohort study in Taiwan. Clin Ther 32: 1294-1303.

17. Chang SS, Hu HY. (2013) Helicobacter pylori is not the predominant etiology for liver cirrhosis patients with peptic ulcer disease. Eur J Gastroenterol Hepatol 25: 159-165.

18. Gisbert JP, Calvet X (2009) Review article: Helicobacter pylori-negative duodenal ulcer disease. Aliment Pharmacol Ther 30: 791-815.

19. Tseng GY, Lin HJ, Fang CT, Yang HB, Tseng GC, et al. (2007) Recurrence of peptic ulcer in uraemic and non-uraemic patients after Helicobacter pylori eradication: a 2-year study. Aliment Pharmacol Ther 26: 925-933.

20. Jung R, MacLaren R (2002) Proton-pump inhibitors for stress ulcer prophylaxis in critically ill patients. Ann Pharmacother 36: 1929-1937.

21. Luo JC, Leu HB, Hou MC, Huang CC, Lin HC, et al. (2012) Cirrhotic patients at increased risk of peptic ulcer bleeding: a nationwide population-based cohort study. Aliment Pharmacol Ther 36:542-550. 